\title{
A General Analysis of Resource Allocation by Competing Individuals
}

\author{
Leslie A. ReAl \\ Department of Zoology, University of Michigan, Ann Arbor, Michigan 48104
}

Received November 14, 1973

\begin{abstract}
The analysis presented in this paper is constructed to determine the pattern of resource allocations among competing individuals of the same and/or different species in some defined habitat. Competing individuals have at their disposal various activities which can be run at different intensities and consume or produce resources in the habitat linearly. The linearity of resource utilization restricts the analysis to exploitative competition. Each resource is assigned values representing the reproductive gain and reproductive cost to the organism incurred while utilizing the resource. The organisms attempt to choose the amounts and kinds of resources which maximize the total reproductive gain. Resource utilization is restricted by the availability of resources in the habitat, the patterns of consumption associated with the activities of the individuals, and the demand for the individual resources (fixed through competition pressure) of the habitat. It is proved that under these restrictions a competitive equilibrium exists where resources are utilized so as to maximize individual reproductive gain and that this equilibrium generates the most efficient utilization of the resources in that habitat. The selection of a diet subject to nutritional requirements is given as an example of possible applications of this approach. The assumptions and restrictions associated with this technique of analysis are discussed.
\end{abstract}

Within recent years many ecologists have become interested in the analysis of resource allocation by individuals of a particular species and by sets of species co-occurring in a defined habitat. Concurrently, the problem of optimal food choice by animals has been a theme of major interest in the ecological and behavioural literature (e.g. Emlen, 1966, 1968; Fretwell, 1972; Marten, 1973; Schoener, 1971). Proposed models of optimal food choice have been concerned with the maximization of energy (caloric) input to the organism, but have neglected general constraints operating on the individual, e.g., nutritional requirements. On the other hand, models of resource allocation among species have been limited to small numbers of species competing for a few resources. This note is intended to present a general model of the allocation of 
large numbers of resources between several individuals of the same and/or different species subject to defined constraining conditions.

Individuals associated with a particular habitat have at their disposal a number of ecological activities which they can operate at various levels or intensities. Individuals may be involved with such diverse activities as hunting, food storage, establishment of territory, nest building, etc. Suppose that the reproductive gain from operating an activity at unity level can be determined, and suppose that the individual seeks to maximize its total reproductive gain subject to certain limitations on the amounts of supplies available as inputs to the activities. In a more formal sense, suppose that an individual performs $n$ activities $G_{1}, \ldots, G_{n}$ and has at its disposal $m$ resources $R_{1}, \ldots, R_{m}$. The activity matrix, $\mathbf{A}$, for the individual is given by the $n \times m$ matrix:

$$
\begin{aligned}
& \begin{array}{lll}
R_{\mathrm{L}} & \cdots & R_{m}
\end{array} \\
& \dot{G}_{1}\left[\begin{array}{ccc}
a_{11} & \cdots & a_{1 m} \\
\vdots & & \vdots \\
a_{n 1} & \cdots & a_{n m}
\end{array}\right]=\mathbf{A} \text {, }
\end{aligned}
$$

where $a_{i j}$ is the amount of $R_{j}$ consumed when $G_{i}$ is operated at unit intensity. An activity $G_{i}$ is being operated at intensity $x_{i}$ if the total consumption of resources is given by the numbers $x_{i} a_{i 1}, x_{i} a_{i 2}, \ldots, x_{i} a_{i n}$. For this discussion we define the supply vector, $\mathbf{S}$, as the vector of amounts of resources available for consumption in the habitat.

Assume there are $q$ individuals in the habitat, where the $k$ th individual can be represented by an activity matrix $\mathbf{A}_{k}$. Our main problem will be to determine how the resources of the habitat are distributed among the component individuals. We will show that this problem is solved through the interactions of individuals in competition for resources in limited supply.

It is convenient to subdivide what we have loosely called resources into two categories: resources such as raw materials which the individuals compete for, and co-resources such as the available equipment which is rigidly attached to a given individual. For Escherichia coli glucose belongs to the first category while the enzymes of the glycolytic pathway belong to the second. In the establishment of territory in birds, space and nesting materials would be resources and associated visual and vocal displays would be co-resources. The category to which an item belongs may depend upon the particular application. For instance, for some purposes one may wish to think of mycorrhizal fungi as being rigidly attached to certain plants and carrying out fixed activities; for others one may assume that plants are competing for the symbiotic relation afforded by the fungi, or visa versa.

It is easy to introduce this distinction between supply types into the model. One method of doing this would be to divide the supply vector into those 
elements indicating resources and those indicating co-resources. An equivalent and notationally more convenient method is to split the activity matrix as well as the supply vector into two parts. Let us first consider the case of co-resources.

Let $\mathbf{X}_{k}=\left(x_{1 k}, x_{2 k}, \ldots, x_{n k}\right)$ be the intensity vector for the $k$ th individual. This vector is limited by the available co-resources according to the inequality:

$$
\mathbf{X}_{k} \mathbf{B}_{k} \leqslant \mathbf{b}_{k},
$$

where $\mathbf{b}_{k}$ is a vector giving the amounts of various sorts of co-resources available to the $k$ th individual. The $i j$ th element of the matrix $\mathbf{B}_{k}$ gives the amount of the $j$ th co-resource used to operate the $i$ th activity at unit level. Note, both $\mathbf{B}_{k}$ and $\mathbf{b}_{k}$ are nonnegative.

Subject to condition (1) above the $k$ th individual consumes or produces resources in a linear manner. That is to say, there is a matrix $\mathbf{A}_{k}$ such that at intensities $\mathbf{X}_{k}$ the individual consumes (or produces) the vector of resources $\mathbf{X}_{k} \mathbf{A}_{k}$. The matrix $\mathbf{A}_{k}$ need not be nonnegative and we shall choose our signs so that a positive coordinate of $\mathbf{X}_{k} \mathbf{A}_{k}$ corresponds to an amount consumed; a negative coordinate to an amount produced. It is easy to see how individuals may be actively producing resources if we consider symbiotic relationships occurring in the biotic community.

There is now the important further constraint which states that the aggregate of all the individuals must not use up more resources than the habitat can provide. Let $\mathbf{S}-\left(\sigma_{1}, \sigma_{2}, \ldots, \sigma_{m}\right)$ be the vector such that $\sigma_{j}$ is the maximum amount of the $j$ th resource which is available in the habitat. Then the desired condition takes the form:

$$
\sum_{k=1}^{q} \mathbf{X}_{k} \mathbf{A}_{k} \leqslant \mathbf{S}
$$

Returning now to the resource allocation problem we see that we are looking for a set of vectors $\mathbf{X}_{k}$ which satisfy the inequalities (1) and (2). As yet no objective function has been introduced, and, in general, there will be an infinite number of solutions to these inequalities. We shall now describe how competition could act to determine such a solution.

In any community, organisms will be limited in one form or another by some primary factor (or the interaction of factors) of production (Liebig's Law of the Minimum). Suppose that an arbitrary nonnegative set of values $P=\left(\pi_{1}, \pi_{2}, \ldots, \pi_{m}\right)$ has been assigned to the various resources of the habitat indicating the reproductive cost (i.e., the amount to which reproductive success is reduced) by utilizing one unit of resource $j, \pi_{j}$. Let $\Psi_{i}^{k}$ be the reproductive gain to the $k$ th individual from operating the $i$ th activity at unit level. Then the gross reproductive gain from operating all activities at levels given by the vectors $\mathbf{X}_{k}$ is $\mathbf{X}_{k} \mathbf{C}_{k}$ where $\mathbf{C}_{k}-\left(\Psi_{\mathbf{1}}{ }^{k}, \Psi_{z^{k}}, \ldots, \Psi_{n}{ }^{k}\right)$. The reproductive cost of 
this operation is given by $\mathbf{X}_{k} \mathbf{A}_{k} \mathbf{P}$. The individual will try to act so as to maximize its reproductive gain given by the expression $\mathbf{X}_{k}\left(\mathbf{C}_{k}-\mathbf{A}_{k} \mathbf{P}\right)$. In other words, given the value vector $\mathbf{P}$ the $k$ th individual acts so as to solve the maximum problem of finding a nonnegative vector $\mathbf{X}_{k}$ such that

$$
\mathbf{X}_{k}\left(\mathbf{C}_{k}-\mathbf{A}_{k} \mathbf{P}\right)
$$

is a maximum subject to condition (1).

However, if each of the $q$ individuals acts so as to solve the above maximization problem the vectors $\mathbf{X}_{k} \mathbf{A}_{k}$ obtained will in general not satisfy condition (2), that is, the utilization of resources demanded by the various individuals may not be compatible with the available rcsources. Classical ccological theory asserts, according to Liebig's Law, that the reproductive costs of the limiting factors will increase as these factors become more limiting. These changes will result in a shift in the intensity vector $\mathbf{X}_{k}$ and the value vector $\mathbf{P}$. Hopefully after a series of such adjustments equilibrium values for reproductive costs will be found such that there is no excess demand for any resource. An example might be in order at this moment.

Consider an organism or group of organisms in some habitat limited by some nutritional requirement, say water requiring rodents in a desert environment. We can see that the organisms expend a certain amount of energy in finding and utilizing foods so as to maintain water balance. This energy cost will be a function of the availability of water in the habitat. This availability will be related to the abundance of water containing foods and the magnitude of competition between individuals over those foods. It is well known that rodents show diet shifts in arcas of specics overlap (Alcoze and Zimmcrman, 1973; Drickamer, 1970; Reichman, 1973). We can imagine that even if the resource is more abundant than some other resources in the habitat (within some definite range), if those other resources are under less demand (the magnitude of competition thereby being lowered) their availability to individuals in the habitat will be greater and we can expect to see a greater representation of those foods in the diets of the animals.

Assume also that there is a very large quantity of magnesium salts in the foods of the habitat. In this case, little if any energy will be expended in utilizing magnesium in the diet. Thus, the value for $\pi$ water will be very high; while $\pi$ magnesium will be comparatively low.

Organisms are well known to exhibit sampling programs and shifts in diet associated with shifts in availability of food items (Allen and Clark, 1962; Gibb, 1962; Goss-Custard, 1970; Moment, 1962; Popham, 1941, 1942; Royama, 1970; Tinburgen, 1960; Whitaker, 1966). We expect these shifts in resource utilization after sampling to be sensitive to the magnitude of competition with high cost, low gain resources being phased out of use. The vector $\mathbf{X}_{k}$ indicating the intensity at which the various activities use the resources is 
sensitive to the values of vector $\mathbf{P}$ using high-cost, low-again resources less often. Since these items will be used less often there will be a decrease of competition over these resources, thus causing shifts in the values of $\mathbf{P}$. As was already pointed out, after a series of such adjustments equilibrium values for reproductive costs can be found. The distribution of resources induced by these quilibrium values is the solution of the allocation problem. We can use linear programming theory to prove the existence of such values; whether they are ever reached in nature remians to be tested.

There is one additional requirement to be made on the equilibrium values. We have remarked that by Liebig's Law the value or reproductive cost of limiting resources will increase. We can use the same law to state that if a resource is over-abundant, then its reproductive cost will drop and approach zero. Thus we require that the equilibrium value of all over-abundant resources be zero. We can now say that a set of $m+1$ vectors $\left(\mathbf{X}_{1}, \mathbf{X}_{2}, \ldots, \mathbf{X}_{m}, \mathbf{P}\right)$ is said to yield a competitive equilibrium if they satisfy conditions (1), (2), and (3) and in addition

$$
\sum_{k=1}^{q} \mathbf{X}_{k} \mathbf{A}_{k} \mathbf{v}_{j}<\sigma_{j}
$$

implies $\pi_{j}=0$, where $\mathbf{v}_{j}$ is the unit vector for the $j$ th coordinate.

Before proving the existence of a competitive equilibrium we make the important ecological observation that if an equilibrium exists, resources will be distributed in such a way as to maximize the total gross reproductive input to the community. Formally we have the following.

Theorem 1. If $\left(\mathbf{X}_{1}, \mathbf{X}_{2}, \ldots, \mathbf{X}_{m}, \mathbf{P}\right)$ yields a competitive equilibrium the vectors $\mathbf{X}_{k}$ maximize the function $\sum_{k=1}^{q} \mathbf{X}_{k} \mathbf{C}_{k}$ subject to conditions (1) and (2). have

Proof. Let $\mathbf{X}_{k}{ }^{\prime}$ be any of vectors satisfying (1) and (2). Then from (3) we

$$
\mathbf{X}_{k}\left(\mathbf{C}_{k}-\mathbf{A}_{k} \mathbf{P}\right) \geqslant \mathbf{X}_{k}{ }^{\prime}\left(\mathbf{C}_{k}-\mathbf{\Lambda}_{k} \mathbf{P}\right), \quad \text { for all } k \text {. }
$$

Summing these inequalities over $k$ gives

$$
\sum_{k=1}^{q} \mathbf{X}_{k} \mathbf{C}_{k}-\sum_{k=1}^{q} \mathbf{X}_{k}{ }^{\prime} \mathbf{C}_{k} \geqslant \sum_{k=1}^{q} \mathbf{X}_{k} \mathbf{A}_{k} \mathbf{P}-\sum_{k=1}^{q} \mathbf{X}_{k}{ }^{\prime} \mathbf{A}_{k} \mathbf{P} .
$$

From (2) we know that $\sum_{k=1}^{q} \mathbf{X}_{k}{ }^{\prime} \mathbf{A}_{k} \leqslant \mathbf{S}$ so $\sum_{k=1}^{q} \mathbf{X}_{k}{ }^{\prime} \mathbf{A}_{k} \mathbf{P} \leqslant \mathbf{S P}$. But from (2) and (4) it easily follows that $\sum_{k=1}^{q} \mathbf{X}_{k} \mathbf{A}_{k} \mathbf{P}=\mathbf{S P}$. Therefore, the right-hand side of $(5)$ is nonnegative and hence:

$$
\sum_{k=1}^{q} \mathbf{X}_{k} \mathbf{C}_{k} \geqslant \sum_{k=1}^{q} \mathbf{X}_{k}{ }^{\prime} \mathbf{C}_{k} .
$$


This result is quite similar to MacArthur (1972) and Gill's (1972) conclusions on exploitative competition. That is, under exploitative competition, utilization of resources within the community will be maximally efficient. The assumptions of linear resource utilization make the present model similarly one of exploitative rather than interference competition. We shall now prove the existence of a competitive equilibrium.

THEOREM 2. If the total gross reproductive input to the community is bounded above, then there exists a competitive equilibrium. (Note that this condition is always satisfied since a perfectly reproducing system is bounded by the total energy entering the system.)

Proof. Consider the maximum problem of finding nonnegative vectors $\mathbf{X}_{k}$ such that

$$
\sum_{k=1}^{q} \mathbf{X}_{k} \mathbf{C}_{k}
$$

is a maximum subject to conditions (1) and (2). Since (6) is bounded above it attains its maximum for some set of vectors $\overline{\mathbf{X}}_{k}$, and therefore from the duality theorem of linear programming (see Karlin, 1959) there exists nonnegative vectors $\overline{\mathbf{Y}}_{1}, \ldots, \overline{\mathbf{Y}}_{m}$ and $\mathbf{P}$ such that

$$
\mathbf{B}_{k} \overline{\mathbf{Y}}_{k}+\mathbf{A}_{k} \mathbf{P} \geqslant \mathbf{C}_{k}, \quad \text { for all } k,
$$

and

$$
\sum_{k=1}^{q} \overline{\mathbf{Y}}_{k} \mathbf{b}_{k}+\mathbf{P S}=\sum_{k=1}^{q} \overline{\mathbf{X}}_{k} \mathbf{C}_{k}
$$

We assert that the vectors $\overline{\mathbf{X}}_{k}$ and $\mathbf{P}$ give the desired equilibrium. To show this we must verify that $\overline{\mathbf{X}}_{k}$ satisfies (3). That is, each $\overline{\mathbf{X}}_{k}$ maximizes $\mathbf{X}_{k}\left(\mathbf{C}_{k}-\mathbf{A}_{k} \mathbf{P}\right)$ subject to (1) above. The dual of this problem is that of finding a nonnegative vector $\mathbf{Y}_{k}$ such that $\mathbf{Y}_{k} \mathbf{b}_{k}$ is a minimum subject to

$$
\mathbf{B}_{k} \mathbf{Y}_{k} \geqslant \mathbf{C}_{k}-\mathbf{A}_{k} \mathbf{P} \text {. }
$$

Now the vector $\overline{\mathbf{X}}_{k}$ is clearly feasible for this problem, and from (7) we see that $\overline{\mathbf{Y}}_{k}$ satisfies (9) and is therefore feasible for the dual problem. By the optimality criterion $\overline{\mathbf{X}}_{k}$ will be optimal if

$$
\overline{\mathbf{Y}}_{k k} \mathbf{b}_{k}=\overline{\mathbf{X}}_{k}\left(\mathbf{C}_{k}-\mathbf{A}_{k} \mathbf{P}\right) .
$$

To prove (10) note that $\overline{\mathbf{Y}}_{k} \mathbf{b}_{k} \geqslant \overline{\mathbf{X}}_{k}\left(\mathbf{C}_{k}-\mathbf{A}_{k} \mathbf{P}\right)$ or

$$
0 \leqslant \overline{\mathbf{Y}}_{k} \mathbf{b}_{k}+\overline{\mathbf{X}}_{k} \mathbf{A}_{k} \mathbf{P}-\overline{\mathbf{X}}_{k} \mathbf{C}_{k}, \quad \text { for all } k .
$$


Summing (11) over all $k$ gives

$$
0 \leqslant \sum_{k=1}^{q} \overline{\mathbf{Y}}_{k} \mathbf{b}_{k}+\sum_{k=1}^{q} \overline{\mathbf{X}}_{k} \mathbf{A}_{k} \mathbf{P}-\sum_{k=1}^{q} \overline{\mathbf{Y}}_{k} \mathbf{C}_{k}
$$

Now from (2) the right-hand side above is at most $\sum_{k=1}^{q} \overline{\mathbf{Y}}_{k} \mathbf{b}_{k}+\mathbf{P S}-\sum_{k=1}^{q} \overline{\mathbf{X}}_{k} \mathbf{C}_{k}$, but from (8) this expression is zero. It follows that equality holds in (12). But the right-hand side of (12) is the sum of the nonnegative terms on the right-hand side of (11). Therefore, each such term is zero, which gives the desired equation (10). Finally, we note that the vectors $\overline{\mathbf{X}}_{k}$ and $\mathbf{P}$ satisfy condition (4), for this condition is precisely the statement of the equilibrium theorem applied to constraints (2).

In summary, under the assumption of linear resource utilization (valid under the restriction to exploitative competition) and subject to the limitations of resource availability within the habitat, an equilibrium point exists which results in maximal resource utilization within the habitat. This equilibrium point is reached through competition for limiting resources and each individual in the habitat trying to maximize its own reproductive gain.

As the simplest example of the biological applications of this approach, let us cxamine the selection of a dict by an organism not in competition with other organisms for food items (i.e., the magnitude of competition is zero and all foods are available according to their absolute abundance).

Many behavioral ecologists have speculated on mechanisms of optimal food choice in animals (Emlen, 1966, 1968; MacArthur and Pianka, 1966; Marten, 1973; Rapport, 1971; Westboy, 1974). Suppose our animal under investigation is presented with $n$ different foods in the wild. Call these $n$ foods $F_{1}, F_{2}, \ldots, F_{n}$. From these $n$ foods the animal must choose a diet subject to satisfying the conditions of the general allocation of resource problem. In general, organisms have a certain set of nutrient requirements which must be incorporated into the diet. Let the nutrients be denoted by $N_{1}, N_{2}, \ldots, N_{m}$. Suppose that our animal is required to consume at least $\lambda_{1}$ units of $F_{1}, \lambda_{2}$ units of $F_{2}, \ldots, \lambda_{m}$ units of $F_{m}$, per day. Let $\beta_{i j}$ denote the amount of the $i$ th nutrient contained in one unit of the $j$ th food. Then we see that the diet must satisfy the nutritional requirements

$$
\sum_{j=1}^{n} x_{j} \beta_{i j} \geqslant \lambda_{i}, \quad \text { for all } i=1,2, \ldots, m .
$$

Remember that $\mathbf{X}=\left(x_{i}\right)$ represents the intensity vector of resource utilization.

Let us assume that associated with each food $F_{i}$ there is a fixed reproductive cost. This cost may entail the energy expended during hunting, preparation, digestion, etc. Let $\pi_{j}$ be the total reproductive cost of one unit of food $F_{j}$. The total reproductive cost of the diet is then given by the expression $\sum_{j=1}^{n} x_{j} \pi_{j}$ which we require to be a minimum. In addition, let us assume that we can 
determine the amount of reproductive gain by utilizing one unit of $F_{j}$. Let $\psi_{j}$ denote this amount. The total reproductive gain of the diet is the $\sum_{j=1}^{n} x_{j} \psi_{j}$ which we require to be a maximum. We see that the objective of the optimal food choice problem is to maximize the linear function

$$
f(x)=\sum_{j=1}^{n}\left(\psi_{j}-\pi_{j}\right) x_{j} .
$$

In some cases the nutritional requirements of the organism may be satisfied by any collection of foods and any number of diets are feasible. In this case we may be able to drop the constraints entirely. More realistically we can delimit the number of constraints to only a small collection of inequalities and deal only with them as constraints. For instance, in most animals it may be supposed that the only nutritional constraints are for the $\mathbf{B}$ vitamins and a small number of amino acids. Under these circumstances, all other nutritional constraints can be dropped.

Since it is very difficult to determine the reproductive gains and losses associated with running any particular activity, many authors have analyzed diet problems (as well as other ecological problems) in terms of energetic gains and losses. Although this is mechanistically easier to handle, we must not forget that energetic relationships may not be directly related (or analogous) to reproductive relationships.

However, the present model could certainly be tested by looking at energetic costs and gains in organisms for which we have some knowledge of their nutritional requirements. This diet problem is also extendable into the general competition model by making $\pi_{j}$ variable with competition pressure and by incorporating other activities beside choice of diet. The form of the model would remain the same. Thus, the model is testable in the general case with the kinds of experiments which are beginning to appear in the ecological literature.

Concluding remarks. Three rather striking assumptions are implicit in any linear programming model: (1) the assumption of linearity, (2) the assumption of an equilibrium system, and (3) the assumption of a deterministic system.

Linearity. In all the models we have examined we assumed that the system can be described by anumerating the effects of all the independent components of the system and summing up effects over all the components. In this way we ignore all higher-order interactional effects. We are quite certain, however, that no natural system is completely devoid of higher-order phenomena. Therefore in order to be realistic we must introduce the added complication of nonlinear terms (which show up mathematically as multiplicatives of the various state variables). We now have two alternatives: (1) We assume that all nonlinear terms are small (i.e., all higher-order effects are negligible) and can 
be ignored, giving us a quite adequate linear approximation to the nonlinear system; or (2) we introduce the nonlinear terms and keep them there.

In many natural situations, linear approximations may prove to be quite adequate for our analysis. By keeping to a linear model we have the strong advantage of being able to find analytic solutions to the problems, an occurrence only rarely found in nonlinear systems. Linear models may be useful for heuristic reasons. Since a linear approximation lends itself to easy mathematical analysis, many interesting and important conclusions can be seen that would otherwise been missed in the fog precipitated by the inclusion of nonlinear factors. We may also use the theorems derived from linear approximations as hints to what analogous theorems may be deduced from the nonlinear sister system. Within this framework, I think that linear analysis should not be considered as trivial or uninteresting, but, on the contrary, as a fundamental step in the analysis of more complicated systems.

Alternatively, we stick to analyzing the nonlinear system as best we can. We could do this for a variety of reasons, e.g. the system under examination may have no good linear approximation which at all resembles the nonlinear system, or we may wish to develop and facilitate the use of non-linear mathematical theory, or we may have exhausted the supply of information which we can get out of the linear approximation and wish to move on to more complicated analyses. Whatever the reasons, we can expand our preexisting linear programming theory into the theory of nonlinear programming, a mathematical programming theory designed specifically to handle higher-order interactions. Unfortunately, nonlinear programming has no general theory, and, much like the theory of nonlinear differential equations, can only handle special cases. This being the case we are severely restricted in choosing the form of our nonlinear model. A recent school of mathematicians, the "global analysts," believe they are on the verge of formulating a gencral theory of nonlinear programs. If the global analysts succeed, we will be in a position to analyze biological interactions that would otherwise be intractable.

Equilibrium assumption. In biological systems, the parameters which describe the system are in a continual state of change with respect to time; while in our models we have assumed that these variables attain constant values and remain constant regardless of time. We can, and many have, described biological systems as a set of differential equations. However, we are no longer interested in merely describing the system through time, but in analyzing the system as it changes through time toward some desired end. We immediately see that we may be in trouble. For if the system changes objectives from one time interval to the next, almost no mathematical analysis can be used and the analysis becomes hopelessly complicated. This exact situation exists in economic systems, and the failure of our present mathematical knowledge to handle this economic 
situation was first pointed out by Russell (Scott Gordan, personal communication). As a result of this dilemma, economists may only be able to analyze their systems over short intervals of time, and then the assumption of equilibrium is of no real consequence. Hopefully, biological systems do not operate in a similar fashion, and optimal dynamic strategies can be derived.

Determinism assumption. If we are lucky enough to come up with dynamical systems which adequately describe biological systems, these dynamical systems may be stochastic, i.e., where the state variables become probability functions of time, rather than deterministic, i.e., where the state variables have a fixed value at any instant in time. Our present models are all deterministic. If the probability distributions of the present state variables are dependent upon the probability distributions of the past state variables, that is, where the system "learns" from its past history, then the system is called adaptive. Biological systems seem to be of the adaptive type and are additionally under some forms of regulatory control. It is my belief that biological systems analysis will gain much by applying adaptive control processes with optimality criteria.

\section{REFERENCES}

Alcoze, T. M. and Zimmerman, E. G. 1973. Food habits and dietary overlap of two heteromyid rodents from the mesquite plains of Texas, J. Mammal. 54, 900-908.

Allen, J. A. and Clakke, B. 1968. Evidence for apostatic selection by wild passerines, Nature (London) 220, 501-502.

Drickamer, LeE C. 1970 . Seed preferences in wild caught Peromyscus maniculatus bairdii and Peromyscus leucopus noveboracensis, J. Mammal. 51, 191-194.

Emlen, J. M. 1966. The role of time and energy in food preference, Amer. Natur. 100, 611-617.

EMLEN, J. M. 1968. Optimal choice in animals, Amer. Natur. 102, 385-387.

Fretwell, S. D. 1972. "Populations in a Seasonal Environment," Monographs in Population Biology No. 5, Princeton Univ. Press, Princeton, NJ.

GiBB, JoHN A. 1962. L. Tinbergen's hypothesis of the role of specific search images, Ibis 104, 106-111.

GILL, D. 1972. Intrinsic rate of increase, saturation densities, and competition ability. I. An experiment with Paramecium, Amer. Natur. 106, 461-471.

Goss-Custaro, J. D. 1970. The responses of redshank (Tringa totanus L.) to spatial variations in the density of their prey, J. Anim. Ecol. 39, 91-113.

Karlin, S. 1959. "Mathematical Methods and Theory in Games, Programming, and Economics," Addison-Wesley, Reading, MA.

MacArthur, R. H. 1972. "Geographical Ecology," Harper \& Row, New York.

MacArthur, R. H. and Pianka, E. P. 1966. On optimal use of a patchy environment, Amer. Natur. 100, 603-609.

Marten, G. C. 1973. An optimization equation for predation, Ecology 54, 92-101.

Momoent, G. B. 1962. Reflexive selection: a possible answer to an old puzzle, Science 136, 262-263. 
Popham, E. J. 1941. The variation in the color of certain species of Arctocorisa (Hemiptera: Corixidae) and its signifigance, Proc. Zool. Soc. Lond. A 111, 135-172.

Popнaм, F. J. 1942. Further experimental studies on the selective action of predators, Proc. Zool. Soc. Lond. A 112, 105-117.

RapPORT, D. 1971. An optimizing model of food selection, Amer. Natur. 105, 575-587.

REIChMaN, O. J. 1973. Comparisons made between the diets of desert rodents and available resources. Abstract No. 16, Amer. Soc. Mammal. 53rd Ann. Meeting, Asilomar, California.

Royama, T. 1970. Factors governing the hunting behavior and selection of food by the Great Tit (Parus major L.), J. Anim. Ecol. 39, 619-668.

Schoener, T. W. 1972. Theory of feeding strategies, Ann. Rev. Ecol. § Syst. 2, 369-404.

Tinberger, L. 1960. The natural control of insects in Pinewoods. I. Factors influencing the intensity of predation by songbirds, Arch. Neerl. Zool. 13, 265-343.

Westoby, M. 1974. An analysis of diet selection by large generalist herbivores, Amer. Natur. 108, 290-304.

Whitaker, J. O., JR. 1966. Food of Mus musculus, Peromyscus maniculatus bardii, and Peromyscus leucopus in Vigo County, Indiana. J. Mammal. 47, 473-486. 